\title{
Utilization of Interventional Oncology Treatments in the United States
}

\author{
Sharon W. Kwan, M.D. ${ }^{1}$, Robert K. Kerlan Jr., M.D. ${ }^{1}$, and Jonathan H. Sunshine, Ph.D..$^{2,3}$ \\ ${ }^{1}$ Department of Radiology and Biomedical Imaging, Division of Interventional Radiology, University \\ of California, San Francisco, San Francisco, CA \\ ${ }^{2}$ Research Department, American College of Radiology, Reston, VA \\ ${ }^{3}$ Department of Diagnostic Radiology, Yale University, New Haven, CT
}

\begin{abstract}
Purpose-To report on the recent national trends in utilization of interventional oncology (IO) treatments, assess the use of these techniques relative to the utilization of alternative oncologic treatments, and provide an assessment of which specialties are providing these services.
\end{abstract}

Materials and Methods-The Centers for Medicare and Medicaid Services Physicians/Supplier Procedure Summary Master Files from 2002-2008 and Limited Data Set Standard Analytical Files from 2002-2007 were used to determine utilization rates of ablative therapies and transarterial embolizations for malignant neoplasms and comparable surgical procedures.

Results-In 2008, 10,045 IO treatments were performed in the Medicare population, or 29 per 100,000 Medicare enrollees. IO treatments of the liver constituted the largest component, at 64\%, followed by the kidneys, at $23 \%$. Over a period of six years, growth was seen in all IO procedures except for transarterial embolizations for renal tumors. Radiologists performed $91 \%$ of all IO procedures in 2008.

Conclusions-IO utilization is modest in volume, but growing. IO treatments comprised the majority of oncologic treatments in the liver but only a small part of treatments in the kidneys and lungs. Radiologists are currently providing the vast majority of IO treatments.

\section{INTRODUCTION}

Interventional oncology (IO) is a rapidly growing field which holds the promise of becoming a major component of interventional radiology (IR) practices. Minimally invasive transcatheter and percutaneous treatments are being performed for many types of neoplasms, whether as sole or adjunctive treatment (1-5), as a bridge to or for downstaging prior to organ transplantation (6-9), or as palliative therapy (10-13).

\footnotetext{
C 2010 The Society of Interventional Radiology. Published by Elsevier Inc. All rights reserved.

Corresponding author: Sharon W. Kwan Department of Radiology and Biomedical Imaging 505 Parnassus Ave, M-391 San Francisco, CA 94143 Phone: (650) 814-3688 Fax: (415) 476-0616 sharon.kwan@ radiology.ucsf.edu.

Publisher's Disclaimer: This is a PDF file of an unedited manuscript that has been accepted for publication. As a service to our customers we are providing this early version of the manuscript. The manuscript will undergo copyediting, typesetting, and review of the resulting proof before it is published in its final citable form. Please note that during the production process errors may be discovered which could affect the content, and all legal disclaimers that apply to the journal pertain.

The material in this manuscript has not been presented at an SIR Annual Meeting.
} 
Scant published analysis is available on the current utilization of IO treatments or who is providing these services. Information about utilization can provide guidance for practices and policy makers dealing with issues such as resource allocation. This study evaluates recent national trends in utilization of IO, specifically focusing on the core services of IO: percutaneous tumor ablation and transcatheter embolization. We also assess the use of these new techniques relative to the utilization of alternative oncologic treatments with similar purposes and provide an assessment of which specialties are providing these treatments.

\section{MATERIALS AND METHODS}

Institutional review board approval was not required for this study as the data sources do not involve individually identifiable health information.

For this study, IO treatments are defined as ablative procedures performed via a percutaneous approach plus all transarterial embolization procedures performed for malignant neoplasms. This definition excludes ablations performed with open or laparoscopic approaches, although we do report utilization levels of these procedures for comparative purposes.

The Centers for Medicare and Medicaid Services (CMS) Physicians/Supplier Procedure Summary (PSPS) Master Files from 2002-2008 were used to obtain utilization data for ablative therapies and comparable surgical resections. These files include aggregated claims information for all patients with Medicare Supplemental Medical Insurance (Medicare Part B) who do not receive their Medicare benefits through a Medicare managed care plan (Medicare Advantage). Claims with relevant procedure codes were selected, using the 2008 Current Procedural Terminology ${ }^{\circledR}$ (CPT) (American Medical Association, Chicago, IL). CPT codes for percutaneous, open, and laparoscopic ablations of the liver, kidneys, and lungs were included. CPT codes for comparable open and laparoscopic resection procedures of the liver, kidneys, and lungs were also included.

Codes for radical and partial nephrectomy were included as they represent the most current surgical treatments for localized renal neoplasms (14). In contrast, the code for simple nephrectomy was not included because these procedures are generally reserved for removal of kidneys damaged from non-oncologic causes such as chronic infection, chronic obstruction, or traumatic injury. Codes for pneumonectomies were not included because locoregional treatments such as radiofrequency (RF) ablation are not considered relevant alternatives to such extensive surgeries. Review of the literature finds that bone RF ablation has primarily been described for treatment of benign tumors (e.g. osteoid osteomas) and palliation for pain resulting from osseous metastases (15). Because the alternative treatments for these diseases are broad, including curettage, resection, external beam radiation, and cementoplasty among others, we could not arrive at a well-defined group of surgical codes for comparison. Thus we included the code for RF ablation of the bone to assess overall utilization of IO treatments but no comparable surgical codes were included. Table 1 lists the CPT codes included in this study.

We could not use the PSPS Master Files to analyze utilization of transcatheter therapies for liver and kidney neoplasms because the CPT coding system does not include transcatheter embolization codes specific to individual organs. We instead used the CMS Limited Data Set Standard Analytical Files (LDS-SAF) from 2002-2007. The LDS-SAFs include de-identified, claims-level data for a random 5\% sample of Medicare enrollees. Because the data are not aggregated, each claim includes a CPT code and an International Classification of Diseases, Ninth Revision, Clinical Modification (ICD-9-CM) code. We attempted to capture all bland embolizations, chemoembolizations, and intraarterial radiotherapies of the liver by counting all allowed claims submitted with the CPT code for transarterial embolization and the ICD-9$\mathrm{CM}$ codes for primary and secondary liver neoplasms. We attempted to capture all 
chemoembolizations and bland embolizations of the kidneys by counting all allowed claims submitted with the CPT code for transarterial embolization and ICD-9-CM codes for renal cancer. These data were obtained from Direct Research, LLC (Vienna, VA). Table 2 lists the combinations of CPT and ICD-9-CM codes included for this part of the study. In order to arrive at the total utilization in the entire Medicare population, the total claims from the LDS-SAF were multiplied by 20. Because the LDS-SAF for 2008 is not yet available, estimates for 2008 was computed by calculating the compound annual growth rate (CAGR) for 2002-2007 and multiplying by the volumes in 2007.

Both PSPS Master Files and LDS-SAFs report the self-designated primary specialty of the provider for each claim using one of approximately 100 codes. Before performing our analysis, we reviewed our data and found that providers who self-designated as "diagnostic radiology" provided approximately five times more IO services than those who self-designate as "interventional radiology". In practice, the majority of radiologists providing IO services would likely be considered an interventional radiologist by most definitions. This discrepancy is likely due to carryovers from self-designations which were recorded prior to creation of IR as a separate specialty code in 1991. Therefore, we concluded that the self-assignment as diagnostic versus interventional radiologist is not meaningful in this context and the two specialty codes were grouped together as "radiology". Note that this methodology differs from that of prior authors who have included only those providers who self-designated as IR for assessing utilization of IR services (16).

All data analyses were performed using SAS 9.2 (SAS Institute, Cary, NC) and Microsoft Excel 2007 (Microsoft Corp., Redmond, WA). Percentage distribution of interventional oncology procedures were computed by organ and by performing physician specialty. Linear trends over time were assessed using least-squares regression; trends were considered significant if the $95 \%$ confidence interval for the slope did not include zero. CAGRs were calculated per 100,000 Medicare enrollees for each calendar year. Enrollee numbers were obtained from the CMS (17) and were calculated as the number of enrollees in Medicare Part $\mathrm{B}$ minus the number of enrollees with Medicare Advantage.

\section{RESULTS}

In 2008, 10,045 IO treatment procedures were performed in the Medicare population, or 29 per 100,000 enrollees. Figure 1 shows the relative percentages of the specific IO treatments performed in 2008. IO treatments of the liver made up the largest part, at $64 \%$ of all IO procedures, followed by treatments in the kidney, at $23 \%$. Bone RF ablation made up the smallest fraction, at $3 \%$ of the total. The distribution between ablations and transarterial embolizations was approximately even, representing $51 \%$ and $49 \%$ of all IO procedures, respectively.

Figures 2a-c summarize the relative percentages of IO and comparable surgical oncologic treatment procedures performed in the liver, kidneys, and lungs in 2008. As can be seen, IO treatments comprise the majority of all oncologic treatment procedures in the liver, making up $54 \%$ of all procedures. When ablations performed via open or laparosopic approaches are included with the IO treatments, they represent $64 \%$ of all procedures of the liver. In comparison, IO treatments are a small part of all oncologic treatment performed in the kidneys and lungs, comprising $10 \%$ and $2.2 \%$ of all procedures, respectively.

\section{Growth rates}

Utilization data and CAGR for each IO procedure included in this study are shown in Table 3. From 2002-2008, the number of transarterial embolizations performed for cancers in the liver increased from 5.5 to 13.5 per 100,000 enrollees, representing a CAGR of 16\%. Utilization of 
percutaneous liver RF ablation increased from 2.3 to 4.6 per 100,000 enrollees, for a CAGR of $12 \%$. In contrast, the number of transarterial embolizations performed for renal cancer decreased, from 0.75 to 0.67 per 100,000 enrollee, representing a CAGR of $-2.0 \%$. Growth rates for other IO treatments should not be considered reflective of long-term trends as data are available for at most a few years.

Figures $3 \mathrm{a}$ and $3 \mathrm{~b}$ compare the utilization rates of all (laparoscopic and open) resection procedures, non-percutaneous ablative procedures, percutaneous ablative procedures, and transarterial embolization procedures performed on the liver and kidney. Resection procedures of the liver increased at CAGR of 5.0\% and ablations via open and laparoscopic approaches decreased, with a CAGR of $-0.53 \%$. In comparison, IO treatments in the liver increased with a CAGR of $15 \%$. The utilization of resection procedures of the kidney was essentially constant from 2002-2008, with a CAGR of $0.12 \%$. Ablations of renal neoplasms via open and laparoscopic approaches increased with a CAGR of 3.1\% from 2006-2008. In comparison, IO treatments in the kidneys increased with a CAGR of 34\% from 2006-2008.

\section{Provider specialties}

The number of procedures and relative share of IO procedures performed by each specialty are shown in Table 4. Radiologists performed 98\%, 79\%, 85\%, and 58\% of IO treatment procedures of the liver, kidneys, lungs, and bone, respectively, in 2008. For the four organs combined, radiologists' share of total IO procedures was $91 \%$ in 2008, down slightly from $93 \%$ in 2006. Apparent reductions were seen in radiologists' share of renal embolizations from 2007-2008 and radiologists' share of percutaneous bone RF ablations from 2006-2008 (although figures for the latter may have been affected by coding errors, as discussed below); however, neither apparent trend was statistically significant. A slight decrease was also seen in radiologists' share of percutaneous lung RF ablations in 2008 compared to 2007. For the liver, the organ in which the majority of IO procedures are being performed, radiologists' share of IO procedures stayed stable at 97-98\% between 2002 and 2008.

An additional picture is presented when ablative procedures by all approaches are considered. While radiologists performed the vast majority of percutaneous liver ablations (95\% in 2008), when ablations performed with open or laparoscopic approaches are included, radiologists performed only $58 \%$ of ablations in the liver in 2008 . Similarly with the kidney, when ablations performed with open or laparoscopic approaches are included, radiologists performed only $41 \%$ of all ablations for renal neoplasms in 2008.

\section{DISCUSSION}

At 29 per 100,000 Medicare enrollees, the number of IO treatments currently performed each year is modest relative to all services provided by interventional radiology (16). Using the National Cancer Institute's Surveillance, Epidemiology, and End Results (SEER) data (18) for age and organ specific cancer incidence rates and assuming equivalent utilization rates per cancer case for the non-Medicare population, we estimate that 26,000 IO treatments were performed in the United States in 2008. Assuming 2,500 active interventional radiologists in the United States (19), this represents approximately ten IO procedures per year per interventional radiologist. There will obviously exist variation in the actual procedure volumes for any individual interventionalist and we expect that the provision of these services is concentrated at specialized tertiary care centers.

The data demonstrate that the number of these procedures performed each year is rapidly growing; the number of Medicare claims for IO treatments increased from 2,951 in 2002 to 9,969 in 2008, representing a CAGR of $22 \%$. For the liver, IO treatments now represent over $50 \%$ of all locoregional treatments. For other organs, IO treatments still represent a small 
fraction of the therapeutic treatments for neoplasms. It remains to be seen whether other nascent IO procedures will continue to grow in volume and as a relative share of all treatments in these organs.

One exception to the general growth seen in IO treatments is with transarterial embolizations of the kidneys. The utilization of transarterial embolization for kidney neoplasms fell, with a CAGR of $-2.0 \%$ during our study period. Interestingly, the number of transarterial embolizations performed for liver neoplasms increased dramatically during this same period, with a CAGR of $+16 \%$. These divergent findings suggest that physicians are, to some extent, following evidenced-based practices. Two landmark studies published by Lo et al. (10) and Llovet et al. (11) in 2002 provided Level I evidence supporting the use of transarterial chemoembolization for treatment of unresectable hepatocellular carcinoma. In contrast, while there are some small case series and cohort studies suggesting that embolization for renal cell carcinoma may reduce peri-operative blood loss or symptoms, or prolong survival for patients with advanced disease (20-23), there are no large, controlled studies supporting these findings. Because most preoperative embolizations were performed prior to radical nephrectomies, the declining numbers may also be affected by the recent trend favoring partial over radical nephrectomies (24).

Open ablations were another procedure category showing a notable decrease in utilization. In the liver, open ablations saw a CAGR of $-2.0 \%$ from 2002-2008 and in the kidneys, open ablations had a CAGR of $-9.6 \%$ from 2006-2008. It is likely that these procedures are being replaced by percutaneous or laparoscopic ablations, both of which saw growth during the periods studied. Although both grew, ablations performed percutaneously grew at a more rapid rate than those with performed laparoscopically. As a result, the ratio of ablations performed percutaneously to those performed laparoscopically rose from 0.67 to 1.4 for the liver between 2002 and 2008 and from 0.61 to 1.2 for the kidney between 2006 and 2008.

Radiologists provided the vast majority (over $90 \%$ across all years studied) of IO services. Radiologists performed almost all transarterial embolization procedures for cancers. This was not surprising, given that the other specialists involved in the surgical management of oncology patients do not currently receive training in complex catheter-based techniques. On the other hand, non-radiologists are performing a small but non-trivial portion of percutaneous ablative procedures. This was seen to the largest extent in the kidneys, where urologists performed $35 \%$ of percutaneous cryoablations and $10 \%$ of percutaneous RF ablations in 2008. In more recent years, some apparent increases were seen in non-radiologists' share of IO treatments in the kidneys, lungs, and bone; however, these decreases were not statistically significant and thus may represent random variability in the data.

While radiologists may currently dominate the provision of IO services, considering only IO treatments neglects the possibility of non-radiologists substituting similar treatments via alternative approaches for IO procedures. Taking a broader perspective that includes such substitution, we found that in organs for which open or laparoscopic approaches for ablation exist, radiologists perform only about half of ablations by all approaches. However, in part due to the more rapid increase in percutaneous ablations relative to non-percutaneous ablations, radiologists' shares of all liver and kidney ablations have been increasing over time.

The continued increase in percutaneous IO procedures suggests acceptance of both ablations and embolizations by the oncologic community as an effective therapy. Whether this trend in favor of percutaneous interventions will continue is unknown and will depend on outcomes. Very few randomized controlled trials are available to aid primary care physicians and oncologists in the management of their patients. The appropriate indications for percutaneous ablations in patients with small lung, kidney, and liver (25) tumors have not been established 
with scientific clarity. Moreover, the role of percutaneous tumoricidal therapies in patients with metastatic liver disease has not been rigorously addressed $(26,27)$. As evidence is accumulated regarding outcomes comparing traditional surgical resection versus laparoscopic versus percutaneous ablation, the types of interventions should be modified appropriately.

If percutaneous image guided interventions are shown to have efficacy comparable to competitive alternative procedures, serious challenges will face the radiologist. Patients receiving IO treatments were previously treated by surgical oncologists, urologists and thoracic surgeons. There will be motivation among these practitioners to gain experience in minimally invasive techniques in order to maintain their volume of activity. It is likely that radiologists will need to assume a greater degree of clinical responsibility to maintain their current position as the dominant specialty providing IO treatments. If the increased degree of clinical participation does not occur, competition for these cases with physicians from other disciplines will almost certainly become more intense.

CMS Medicare datasets have the advantage of representing data on over 30 million patients each year, or about one-third of the healthcare workload in the United States. A limitation of these data is that they are predominantly comprised of services provided to the elderly. While the non-elderly population will have different utilization rates, trends are likely to be similar. Additionally, as with all studies utilizing claims data, the results are subject to errors resulting from miscoding. We suspect that some of the unexpected results for the providers of bone RF ablation resulted from billing error because, from our personal experience, anesthesiologists and general practitioners are not generally performing RF ablations. This suspicion is supported by fact that the non-radiologist volumes reported for bone RF ablation are very inconsistent over time. On the other hand, it is also plausible that these practitioners are providing RF ablations, perhaps for pain relief in the case of anesthesiologists. Lacking empirical data to determine which claims represent true claims, we report the data as they were submitted to the CMS. Similar anomalies were not observed for procedure codes other than bone RF ablations.

We are also limited in our assessment of utilization by the availability of CPT codes. CPT codes for ablative procedures are relatively new: the code for bone RF ablation was created in 2004, codes for percutaneous kidney ablations were created in 2006, and the code for lung RF ablation was created in 2007. CPT codes still do not exist for percutaneous ethanol injection or percutaneous cryoablation of the liver. In the years when no relevant CPT code existed, these procedures were probably miscoded under other similar CPT codes or billed as unlisted procedures. The result would be to overestimate the volume of services described by codes under which these procedures were miscoded and to underestimate total IO procedures (because unlisted procedures were not included in our total). We estimate this effect to be small, because if a procedure were being performed at substantial volumes for a multi-year period, specialty societies would likely have successfully applied for the creation of a relevant Category I or III CPT code.

Finally, this study was designed to examine utilization levels based on provider claims data and thus the results should not be misinterpreted to represent the prevalence of various treatment strategies on a population level. Many patients undergo more than one of these procedures and/or the same procedure multiple times during the course of their treatment.

In conclusion, our study finds that IO utilization is still modest, but growing. IO procedures currently represent the majority of locoregional treatments for cancers in the liver, but a small portion of therapeutic treatments for neoplasms in the kidneys and lungs. Ablations performed via a percutaneous approach are increasing at a higher rate than those performed via other approaches. Radiologists are performing nearly all transarterial embolizations and the majority of percutaneous ablations. 


\section{Acknowledgments}

S.W.K. is supported by the National Institute of Biomedical Imaging and Bioengineering Grant NIH/NIBIB T32 EB001631-05.

\section{REFERENCES}

1. Lencioni RA, Allgaier H, Cioni D, et al. Small hepatocellular carcinoma in cirrhosis: randomized comparison of radio-frequency thermal ablation versus percutaneous ethanol injection. Radiology 2003;228:235-240. [PubMed: 12759473]

2. Zhang Y, Liang H, Chen M, et al. Hepatocellular carcinoma treated with radiofrequency ablation with or without ethanol injection: a prospective randomized trial. Radiology 2007;244:599-607. [PubMed: 17641378]

3. Hegarty NJ, Gill IS, Desai MM, Remer EM, O'Malley CM, Kaouk JH. Probe-ablative nephron-sparing surgery: cryoablation versus radiofrequency ablation. Urology 2006;68:7-13. [PubMed: 16857454]

4. Zagoria RJ, Traver MA, Werle DM, Perini M, Hayasaka S, Clark PE. Oncologic efficacy of CT-guided percutaneous radiofrequency ablation of renal cell carcinomas. AJR Am J Roentgenol 2007;189:429436. [PubMed: 17646471]

5. Woertler K, Vestring T, Boettner F, Winkelmann W, Heindel W, Lindner N. Osteoid osteoma: CTguided percutaneous radiofrequency ablation and follow-up in 47 patients. J Vasc Interv Radiol 2001;12:717-722. [PubMed: 11389223]

6. Graziadei IW, Sandmueller H, Waldenberger P, et al. Chemoembolization followed by liver transplantation for hepatocellular carcinoma impedes tumor progression while on the waiting list and leads to excellent outcome. Liver Transpl 2003;9:557-563. [PubMed: 12783395]

7. Belghiti J, Carr B, Greig P, Lencioni R, Poon R. Treatment before liver transplantation for HCC. Ann Surg Oncol 2008;15:993-1000. [PubMed: 18236111]

8. Chapman WC, Majella Doyle MB, Stuart JE, et al. Outcomes of neoadjuvant transarterial chemoembolization to downstage hepatocellular carcinoma before liver transplantation. Ann Surg 2008;248:617-625. [PubMed: 18936575]

9. Yao FY, Hirose R, LaBerge JM, et al. A prospective study on downstaging of hepatocellular carcinoma prior to liver transplantation. Liver Transpl 2005;11:1505-1514. [PubMed: 16315294]

10. Lo C, Ngan H, Tso W, et al. Randomized controlled trial of transarterial lipiodol chemoembolization for unresectable hepatocellular carcinoma. Hepatology 2002;35:1164-1171. [PubMed: 11981766]

11. Llovet JM, Real MI, Montaña X, et al. Arterial embolisation or chemoembolisation versus symptomatic treatment in patients with unresectable hepatocellular carcinoma: a randomised controlled trial. Lancet 2002;359:1734-1739. [PubMed: 12049862]

12. Belfiore G, Moggio G, Tedeschi E, et al. CT-guided radiofrequency ablation: a potential complementary therapy for patients with unresectable primary lung cancer--a preliminary report of 33 patients. AJR Am J Roentgenol 2004;183:1003-1011. [PubMed: 15385294]

13. Goetz MP, Callstrom MR, Charboneau JW, et al. Percutaneous image-guided radiofrequency ablation of painful metastases involving bone: a multicenter study. J Clin Oncol 2004;22:300-306. [PubMed: 14722039]

14. Nguyen CT, Campbell SC, Novick AC. Choice of operation for clinically localized renal tumor. Urol. Clin. North Am 2008;35:645-655. vii. [PubMed: 18992618]

15. Rosenthal DI. Radiofrequency treatment. Orthop. Clin. North Am 2006;37:475-484, viii. [PubMed: 16846772]

16. Khan N, Murphy TP, Soares GM, Zahir IS. Clinical services provided by interventional radiologists to Medicare beneficiaries in the United States, 2000-2003. J Vasc Interv Radiol 2005;16:1753-1757. [PubMed: 16371546]

17. Centers for Medicare \& Medicaid Services. Medicare Trustees Report. 2009

18. National Cancer Institute. Surveillance, Epidemiology, and End Results (SEER) Program, SEER*Stat Database: Incidence - SEER 17 Regs Limited-Use + Hurricane Katrina Impacted Louisiana Cases, Nov 2008 Submission for 2000-2006. [Nov 11, 2009]. Available at http://seer.cancer.gov/statistics/. Published April 2009. 
19. Sunshine JH, Lewis RS, Bhargavan M. A portrait of interventional radiologists in the United States. AJR Am J Roentgenol 2005;185:1103-1112. [PubMed: 16247117]

20. Maxwell NJ, Saleem Amer N, Rogers E, Kiely D, Sweeney P, Brady AP. Renal artery embolisation in the palliative treatment of renal carcinoma. Br J Radiol 2007;80:96-102. [PubMed: 17495058]

21. Schwartz MJ, Smith EB, Trost DW, Vaughan ED. Renal artery embolization: clinical indications and experience from over 100 cases. BJU Int 2007;99:881-886. [PubMed: 17166242]

22. Wallace S, Chuang VP, Swanson D, et al. Embolization of renal carcinoma. Radiology 1981;138:563570. [PubMed: 7465831]

23. Bono AV, Caresano A. The role of embolization in the treatment of kidney carcinoma. Eur Urol 1983;9:334-337. [PubMed: 6653624]

24. Lam JS, Shvarts O, Pantuck AJ. Changing concepts in the surgical management of renal cell carcinoma. Eur Urol 2004;45:692-705. [PubMed: 15149740]

25. Poon D, Anderson BO, Chen L, et al. Management of hepatocellular carcinoma in Asia: consensus statement from the Asian Oncology Summit 2009. Lancet Oncol 2009;10:1111-1118. [PubMed: 19880065]

26. Wong SL, Mangu PB, Choti MA, et al. American society of clinical oncology 2009 clinical evidence review on radiofrequency ablation of hepatic metastases from colorectal cancer. J Clin Oncol 2010;28:493-508. [PubMed: 19841322]

27. Mulier S, Ruers T, Jamart J, Michel L, Marchal G, Ni Y. Radiofrequency ablation versus resection for resectable colorectal liver metastases: time for a randomized trial? An update. Dig Surg 2008;25:445-460. [PubMed: 19212117] 


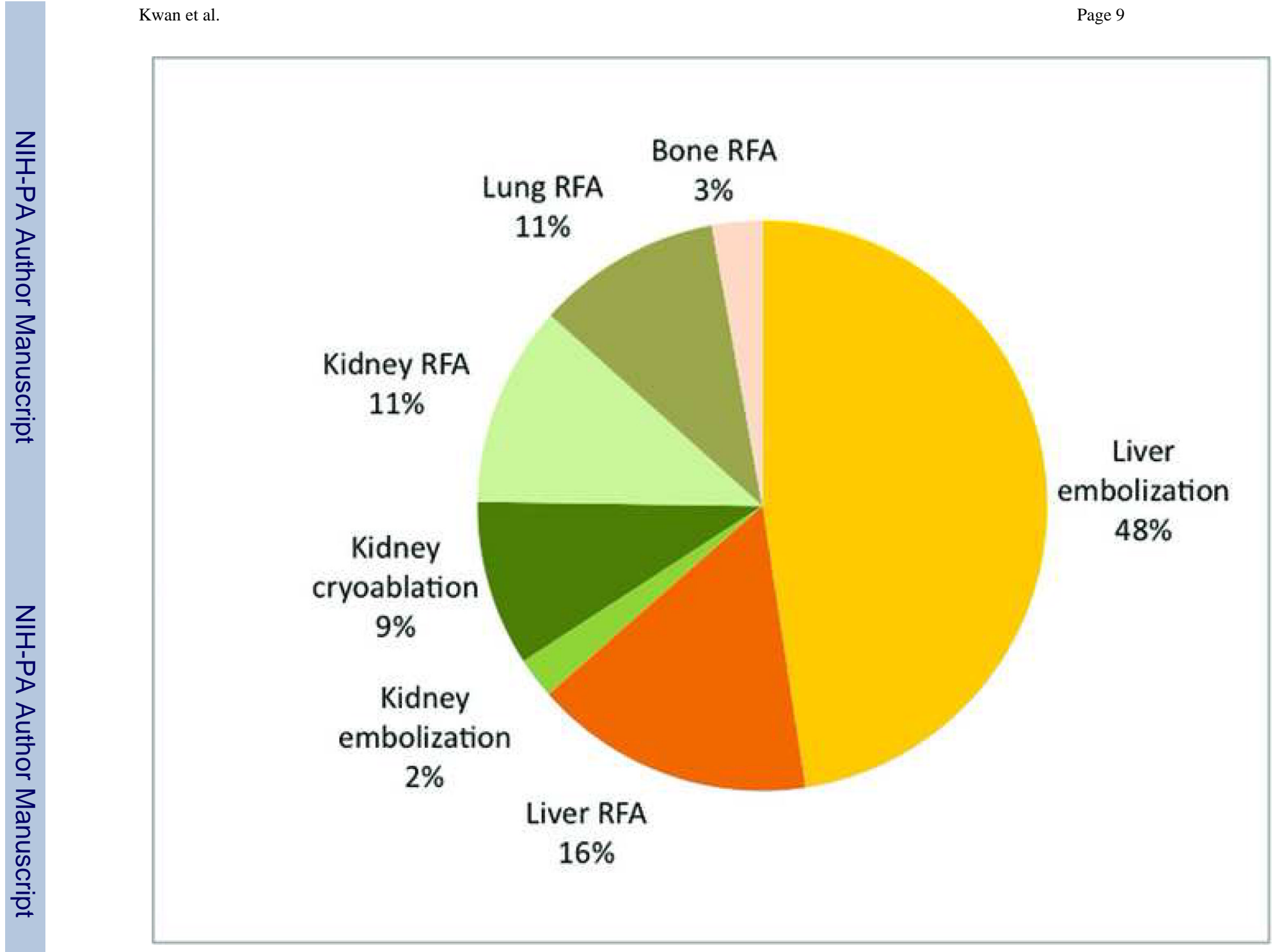

Figure 1.

Various IO procedures as a percentage of all IO procedures performed in 2008. 


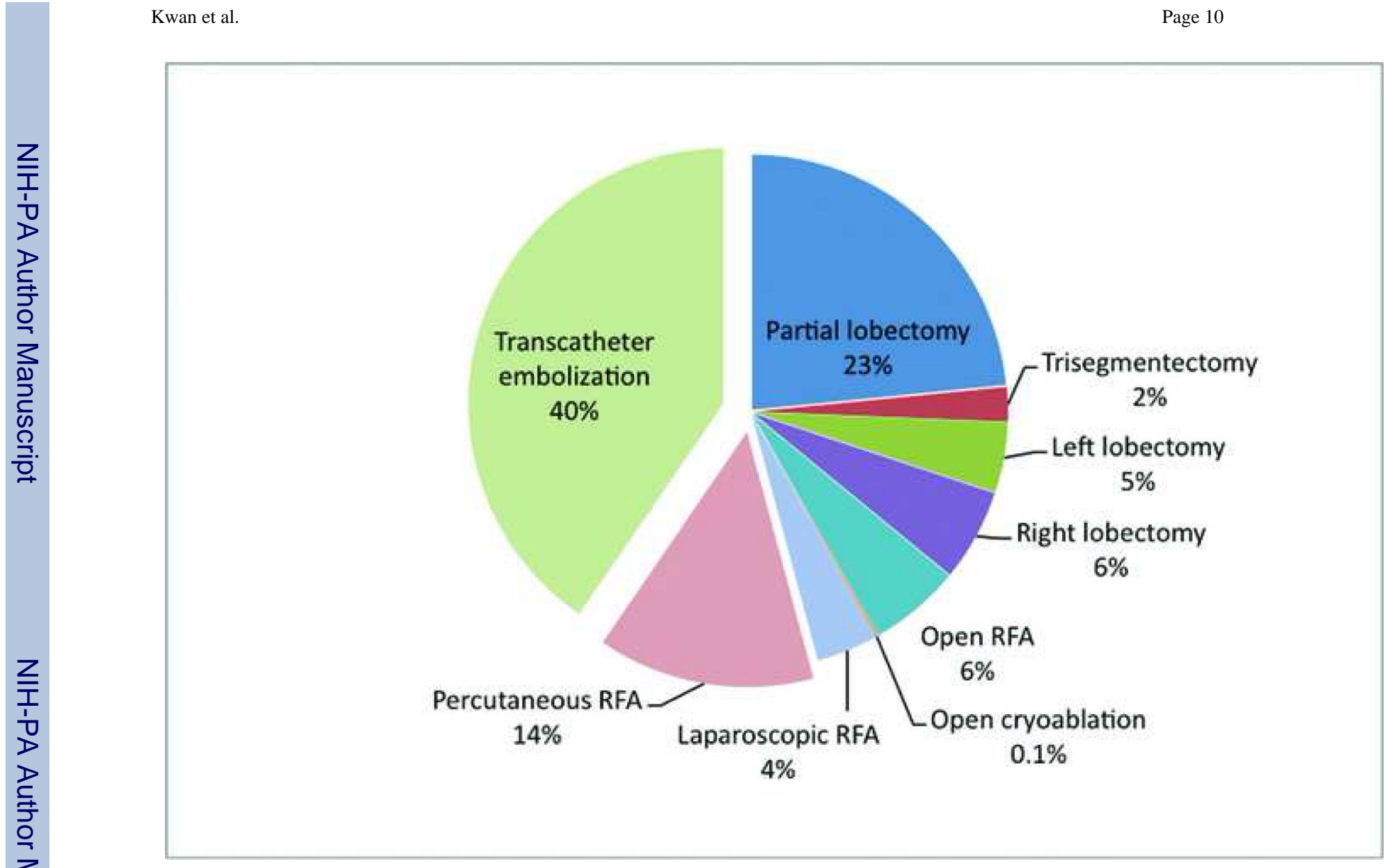

Figure 2a.

Organ-specific procedures as a percentage of all comparable IO and surgical procedures of the liver in 2008. 


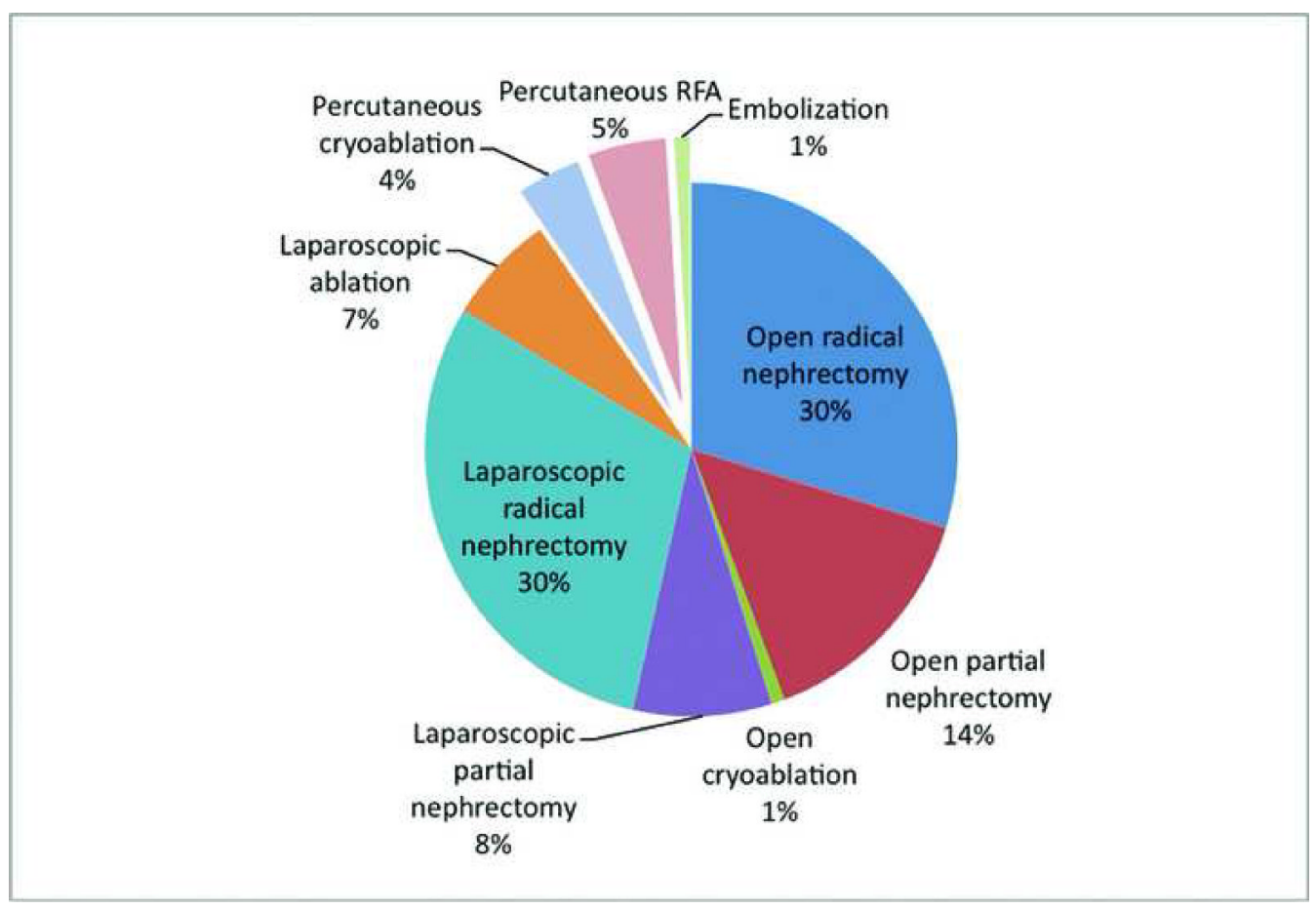

Figure 2b.

Organ-specific procedures as a percentage of all comparable IO and surgical procedures of the kidneys in 2008. 


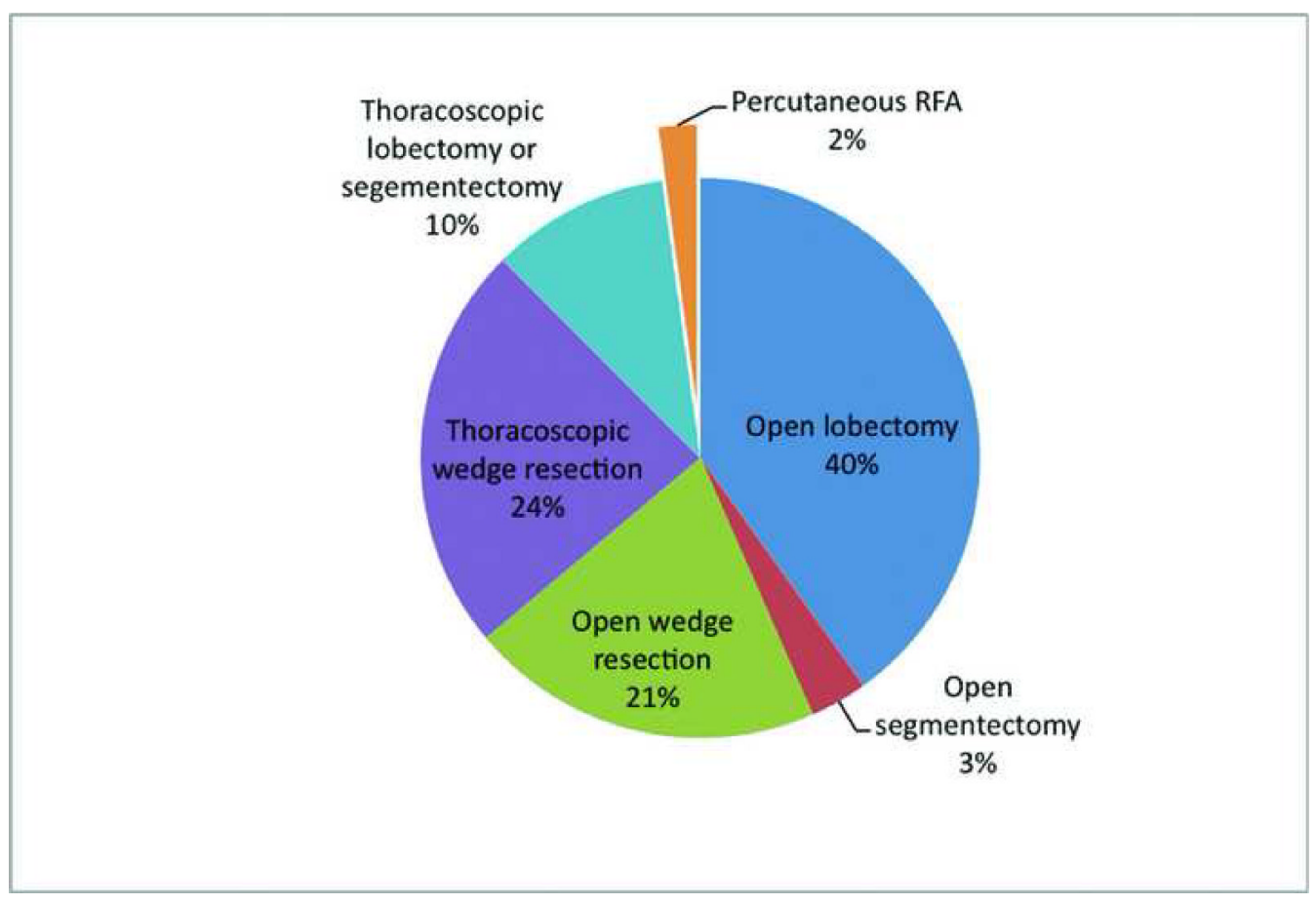

Figure 2c.

Organ-specific procedures as a percentage of all comparable IO and surgical procedures of the lungs in 2008. 


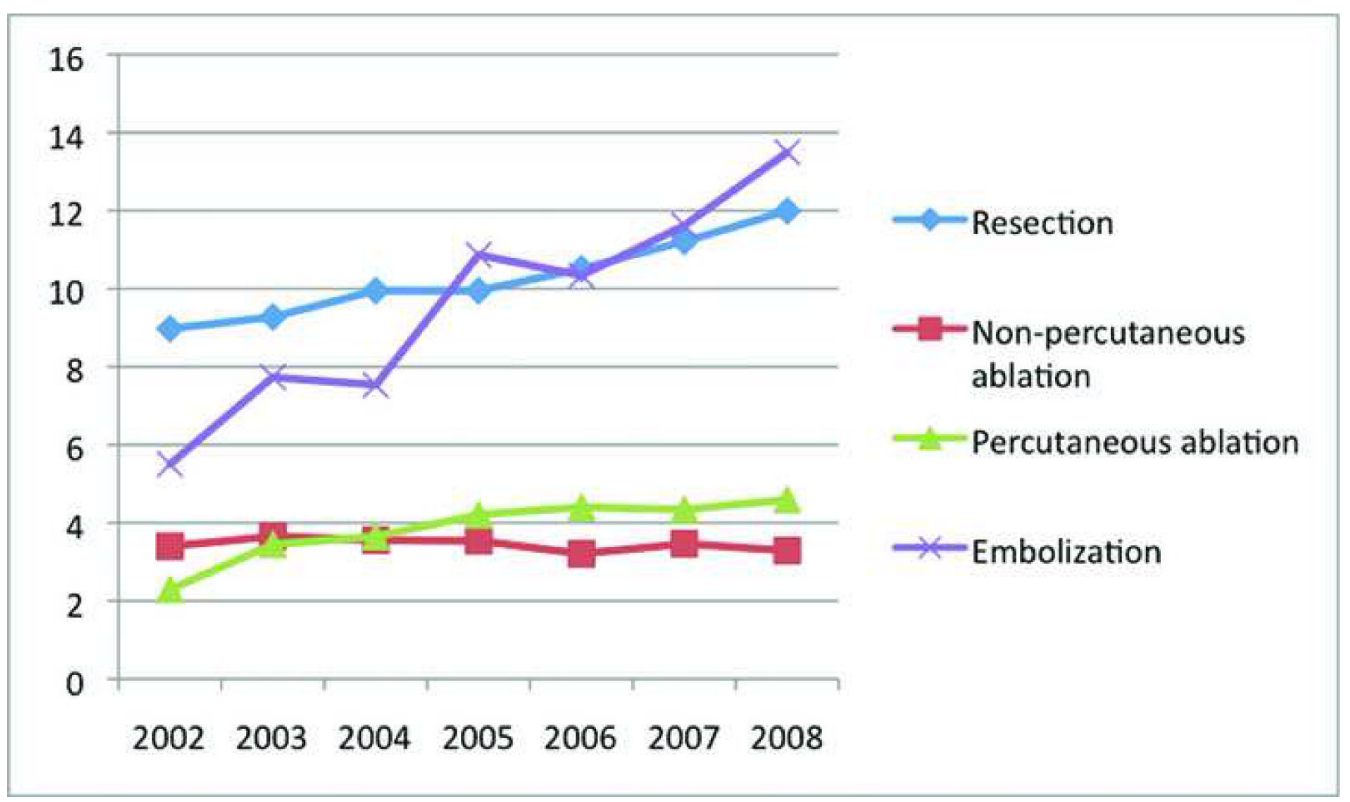

Figure 3a.

Utilization of liver procedures, grouped by procedure type. Numbers are procedures performed per 100,000 Medicare enrollees. 


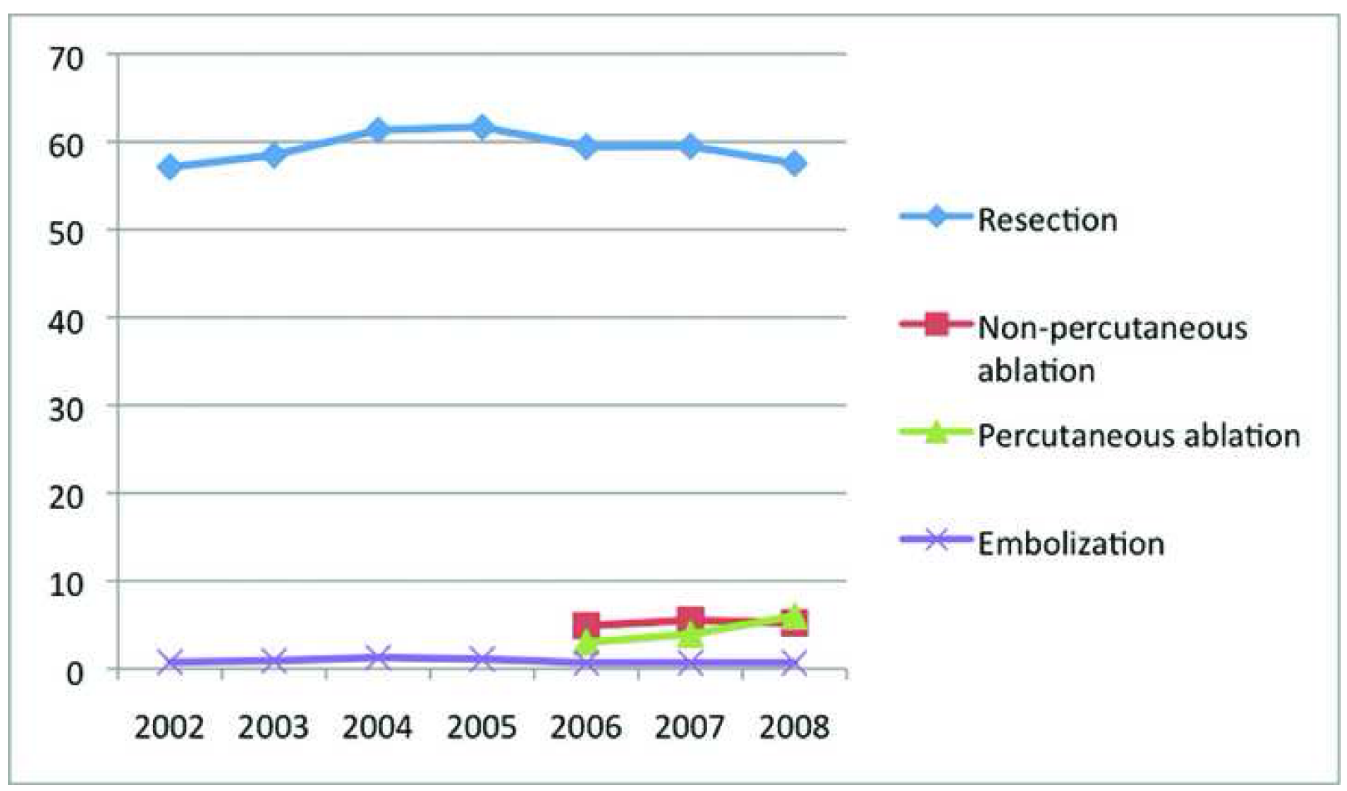

Figure 3b.

Utilization of renal procedures, grouped by procedure type. Numbers are procedures performed per 100,000 Medicare enrollees. Data are not available for ablations prior to 2006 because the relevant CPT codes were created in 2006. 
Table 1

Included CPT codes for ablative treatments and comparable surgical procedures

\begin{tabular}{lll}
\hline Organ & CPT codes & Procedure \\
\hline Liver & 47382 & Percutaneous RFA \\
& 47370 & Laparoscopic RFA \\
& 47380 & Open RFA \\
& 47381 & Open cryoablation \\
& 47120 & Open partial lobectomy \\
& 47122 & Open trisegmentectomy \\
& 47125 & Open left lobectomy \\
\hline Kidney & 57130 & Open right lobectomy \\
& 50592 & Percutaneous RFA \\
& 50593 & Percutaneous cryoablation \\
& $0135 T$ & Percutaneous cryoablation (Category III code pre-2008) \\
& 50542 & Laparoscopic ablation \\
& 50545 & Laparoscopic radical nephrectomy \\
& 50543 & Laparoscopic partial nephrectomy \\
& 50250 & Open cryoablation \\
& 50240 & Open partial nephrectomy \\
\hline Lung & 50230 & Open radical nephrectomy \\
& 32998 & Percutaneous RFA \\
& 32657 & Thoracoscopic wedge resection \\
& 32663 & Thoracoscopic lobectomy \\
32500 & Open wedge resection \\
\hline 32480 & Open lobectomy \\
\hline & 20982 & Open segmentectomy \\
\hline
\end{tabular}




\section{Table 2}

CPT code and ICD-9-CM code combinations used to capture liver and renal embolizations performed for oncologic therapy

\begin{tabular}{llll}
\hline IO procedure captured & CPT code & ICD-9-CM codes and descriptions \\
\hline Liver embolizations = & 37204 PLUS & 155 & Malignant neoplasm of the liver, primary \\
& & 155.2 & Malignant neoplasm of the liver, not specified as primary or secondary \\
& & 197.7 & Malignant neoplasm of the liver, secondary \\
& & 259.2 & Carcinoid syndrome \\
Renal embolizations = & 37204 PLUS & 189 & Malignant neoplasm of the kidney, except pelvis \\
& & 189.1 & Malignant neoplasm of the renal pelvis \\
\hline
\end{tabular}

Note.--CPT code 37204 is for percutaneous transcatheter occlusion or embolization, non-central nervous system, non-head or neck. 


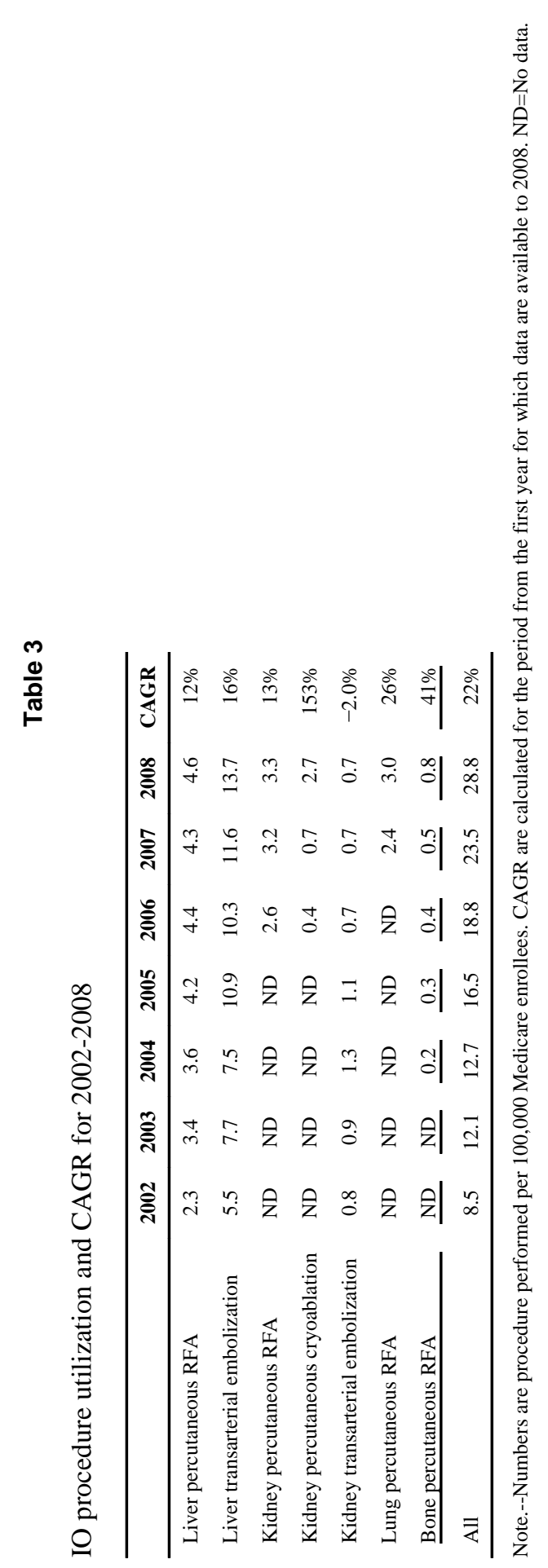

J Vasc Interv Radiol. Author manuscript; available in PMC 2011 July 1. 


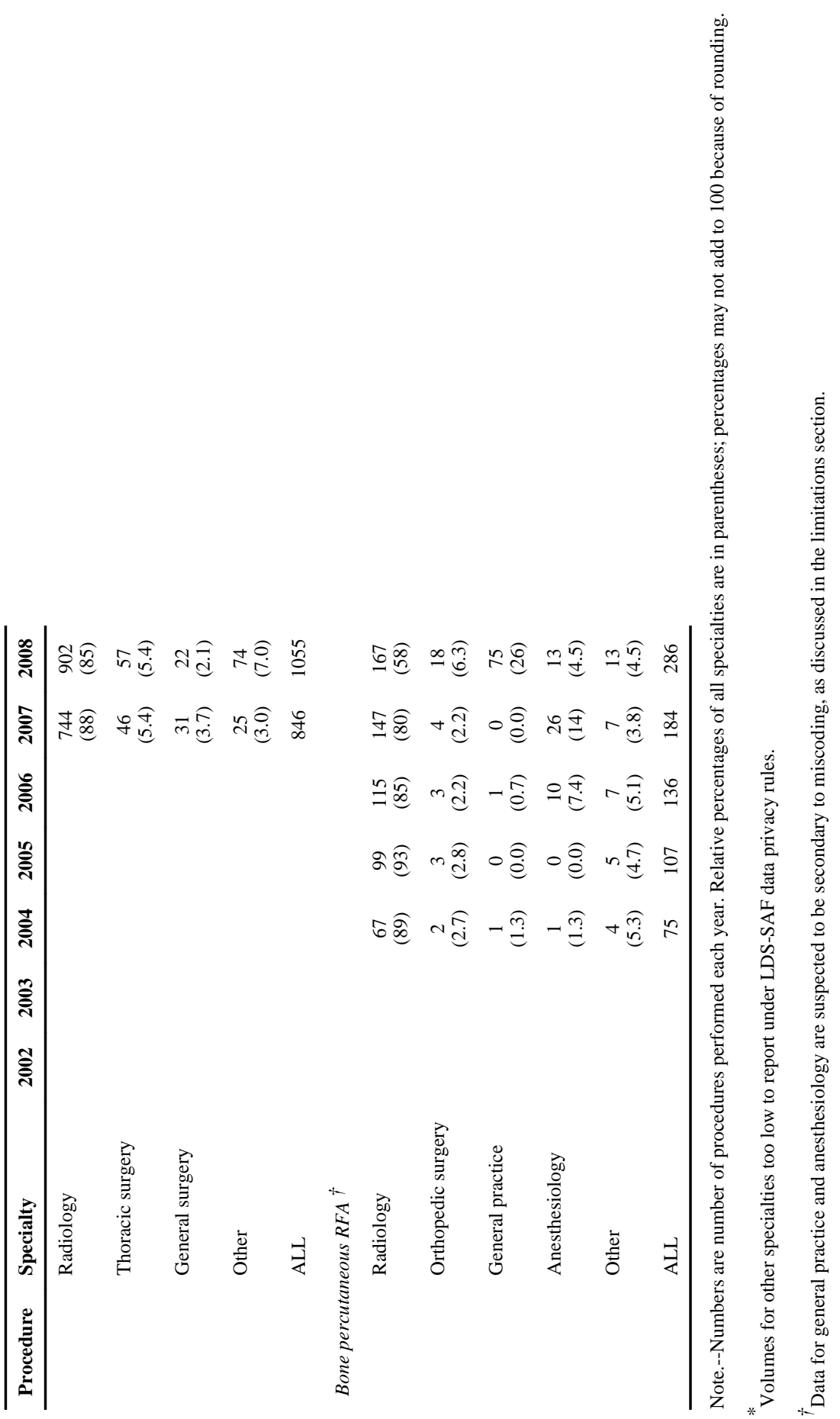

J Vasc Interv Radiol. Author manuscript; available in PMC 2011 July 1. 